\title{
Secondary Hemophagocytic \\ Lymphohistiocytosis, \\ Macrophage Activation \\ Syndrome, and Hyperferritinemic \\ Sepsis-Induced Multiple-Organ \\ Dysfunction Syndrome \\ in the Pediatric ICU
}

\author{
Joseph A. Carcillo, Bita Shakoory, \\ and Leticia Castillo
}

\section{Introduction}

In the past decade, hemophagocytic lymphohistiocytosis (HLH) has been increasingly recognized in critically ill pediatric patients. HLH can be familial or secondary, with most critically ill patients who develop this disease process having the latter. Secondary HLH (sHLH) that occurs in critically ill patients is classically referred to as macrophage activation syndrome (MAS) when triggered by drugs or when it occurs in patients with underlying rheumatologic disease and hyperferritinemic sepsis-induced multiple-organ dysfunction syndrome (MODS) when it is precipitated by infectious etiologies. As awareness of these entities has increased, clinical

J. A. Carcillo $(\bowtie)$

University of Pittsburgh, Department of Critical Care

Medicine, Pittsburgh, PA, USA

e-mail: carcilloja@ccm.upmc.edu

B. Shakoory

PRA Health Sciences, Raleigh, NC, USA

National Institute of Allergy and Infectious Diseases (NIAID), Bethesda, MD, USA

L. Castillo

Pediatric Critical Care, Universidad de Texas Medical

Branch, Galveston, TX, USA

e-mail: Letcasti@UTMB.edu intensivists have been faced with new challenges that have precipitated diagnostic and therapeutic controversies. The need for prompt and aggressive immune-modulating treatment in patients with sHLH requires early recognition and diagnosis. However, clinical and pathologic similarities between sHLH and non-hyperferritinemic sepsis, absence of optimal diagnostic tools in sHLH, and suboptimal understanding of this entity by clinicians often result in delayed or missed diagnoses.

The diagnostic challenges in sHLH are, in part, rooted in the absence of evidence-based diagnostic and therapeutic recommendations for its diagnosis and management. In fact, in most clinical settings, the diagnostic criteria and treatment protocols for familial HLH (Fig. 14.1) are also used in sHLH. In a recent review article however, it was shown that diagnostic criteria used for familial HLH do not differentiate these patients with sHLH, whether it be MAS or hyperferritinemic sepsis-induced MODS [1]. Similarly, treatment protocols for these entities vary, depending on the clinical settings: hematologists treat both familial and sHLH with the highly toxic traditional familial HLH treatment protocols, which include etoposide and dexamethasone, whereas rheumatologists have 


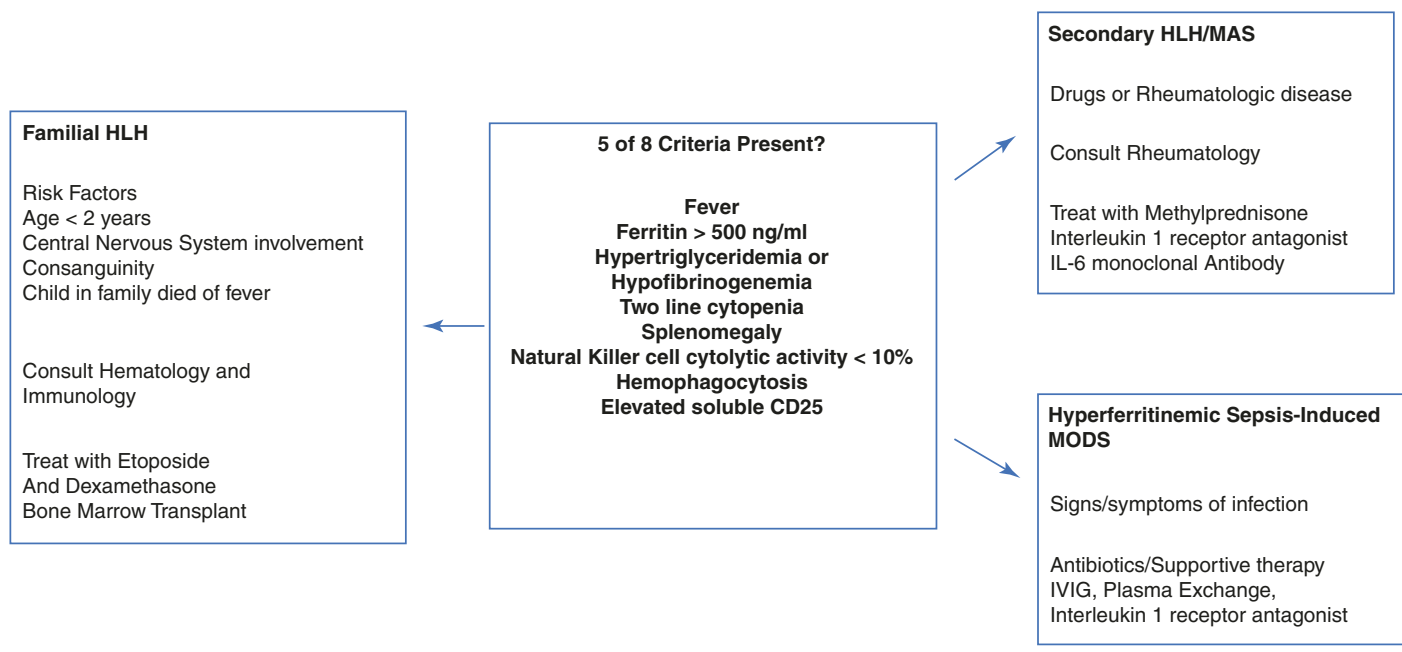

Fig. 14.1 Five of the eight criteria may represent familial hemophagocytic lymphohistiocytosis (HLH), macrophage activation syndrome (MAS), or hyperferritinemic

successfully used less toxic therapies such as pulse-dose corticosteroids (e.g., methylprednisolone) in combination with cytokine-targeted therapies such as anakinra (IL-1 receptor antagonist) or tocilizumab (anti-IL-6 antibody) for MAS. Meanwhile, intensivists who encounter patients with sHLH will most often be faced with hyperferritinemic sepsis-induced MODS and generally advocate source control with antimicrobial therapy and supportive care, but adjunctive therapies such as methylprednisolone, intravenous immunoglobulin (IVIg), and plasma exchange also have a role. Such variations in nomenclature, diagnosis, and therapy emphasize the need for evidence-based data to address the clinical goals of prompt diagnosis and appropriate treatment. The purpose of this chapter is to provide intensivists with a conceptual framework to better understand and navigate these differing management principles in collaboration with their valued colleagues. sepsis-induced multiple-organ dysfunction syndrome (MODS), each of which has a different therapeutic approach

\section{Clinical Vignette 1: Familial Versus Secondary HLH}

A previously healthy infant is admitted to a pediatric ICU after presenting with status epilepticus. The admitting intensivist elicits a history of consanguinity and another child in the family dying from "fever," both of which are risk factors for familial HLH. During the subsequent $48 \mathrm{~h}$ of her hospital course, she develops several clinical criteria for familial HLH (Fig. 14.1). A bone marrow aspirate is obtained, and the infant is started on a familial HLH treatment regimen of dexamethasone and etoposide. The child recovers and is discharged to home.

One month later, genetic analysis confirms the diagnosis. Specifically, it reveals a homozygous UNC13D gene variant 
which leads to ineffective natural killer cell eradication of virus infection and ineffective activated immune cell death. Based on these findings, the child undergoes bone marrow transplantation.

Familial HLH is typically triggered by a viral infection in a host who has a genetically determined inability to induce granzyme-perforin-mediated cytolytic killing. In these children, nonmalignant lymphoproliferation leads to high levels of lymphocyte-derived interferon- $\gamma$ that activates macrophages. Treatment with dexamethasone and etoposide induces lymphocyte apoptosis and reduces macrophage activation. In contrast, secondary HLH is associated with macrophage activation in patients without hereditary predisposition to such a process, and it is typically triggered by severe systemic inflammation such as that occurs in sepsis.

\section{Historical Context}

In 1988, Suster and colleagues reviewed bone marrow, lymph node, and spleen histology from 230 consecutive intensive care unit adult autopsies and identified cases of histiocytic hyperplasia with hemophagocytosis (HHH) [2]. They reported moderate to severe $\mathrm{HHH}$ in 102-230 bone marrow specimens (44\%), 79 of 191 lymph node specimens $(41 \%)$, and 16 of 209 spleen specimens $(8 \%)$. There was a strong blood transfusion dose relationship, with patients who received more than 5 transfusions nearly 60 times as likely to have $\mathrm{HHH}$ as compared to patients who received no transfusions. Bacterial sepsis (adjusted risk odds ratio 4.1) was also independently associated with $\mathrm{HHH}$.

More recently, Strauss and colleagues [3] evaluated 107 consecutive medical ICU patient autopsies and found mild to severe $\mathrm{HHH}$ in 69 (65\%) [3]. The authors similarly found HHH to be associated with sepsis and a number of blood transfusions. Patients with $\mathrm{HHH}$ were significantly less likely to have died due to cardiovascular causes (HHH 32\% versus no HHH 74\%) and more likely to die due to MODS (HHH 39\% versus no $\mathrm{HHH}$ $18 \%$ ), with a characteristic organ failure pattern of elevated bilirubin, liver enzymes, and disseminated intravascular coagulation. Patients with $\mathrm{HHH}$ were also more likely to require catecholamine infusions, mechanical ventilation, and renal replacement therapy. Autopsies with histology graded severe $\mathrm{HHH}$ also had more siderosis suggesting iron overload and more CD8 T cells in the bone marrow suggesting $\mathrm{T}$-cell activation. These two autopsy studies associated HHH with more severe critical illness but could not determine if it was a novel and clinically relevant process or a secondary phenomenon.

Since these seminal reports were published, nomenclature for $\mathrm{HHH}$ has changed, with the aforementioned clinical descriptors now being used for secondary hemophagocytic lymphohistiocytosis ( $\mathrm{HHLH}$ ) - macrophage activation syndrome (MAS) for patients with sHLH precipitated by drugs or rheumatologic disease or hyperferritinemic sepsis-induced MODS, depending on the clinical setting involved [3-7].

\section{Experimental Models of sHLH}

Experimental models provide support for macrophage (i.e., histiocyte) activation associated with sHLH as an important pathway to 
MODS. Steinberg and colleagues developed the sterile model of zymosan (the cell wall of the fungus saccharomyces A) plus mineral oil injected intraperitoneally to induce MODS in rodents [8]. This model results in initial hypovolemic shock followed by persistent macrophage activation. Injection of either zymosan or mineral oil alone does not induce MODS, suggesting the need for a "two-hit" insult of both toll-like receptor (TLR) stimulation by zymosan and unremitting particulate irritation by mineral oil to induce persistent macrophage activation. In another rodent model, Behrens and colleagues reported that repeated (i.e., not single time) TLR9 stimulation with CpG oligodeoxynucleotides transformed an otherwise innocuous endotoxin challenge (TLR4 stimulation) in mice into a MODS model of macrophage activation with cytopenias, splenomegaly, hyperferritinemia, and hepatitis [9]. Similarly, in another murine model of cecal ligation and perforationinduced sepsis, additional $\mathrm{CpG}$ injection induced cytokine production by macrophages and hepatic mononuclear cells, followed by the development of liver injury and MODS-induced mortality [10]. Importantly, the sHLH phenotype elicited with repeated TLR9 stimulation is exacerbated in knockout mice deficient in native hepatic IL-1 receptor antagonist protein production but can be ameliorated by interferon- $\alpha$-induced production of IL-1 receptor antagonist protein or with direct administration of recombinant IL-1 receptor antagonist protein (anakinra) in vivo [11]. In short, liver dysfunction in this model appears to be related in part to IL-1-mediated inflammation [12].

\section{Different Inflammation Pathobiologies in SHLH}

The clinical criteria used to describe the constellation of symptoms and signs indicative of these syndromes are considered to be biomarkers for a state of uncontrolled macrophage and $\mathrm{T}$ lymphocyte inflammation. The NK cell, as the most important "cellular" controller of macrophage and T lymphocyte activation, is considered central to the pathobiology of these conditions. Uncontrolled inflammation can be due in part to ineffective NK cell cytolytic function. Defects or deficiencies in the ability of the NK cell to kill viruses and cancer cells and to turn off the host reticuloendothelial system, macrophage, dendritic cell, and lymphocyte activation can be related to one of the three pathologic conditions: (1) absent NK cell cytolytic activity unrelated to numbers of NK cells present (familial HLH), (2) reduced NK cell cytolytic activity unrelated to numbers of NK cells present (sHLH/MAS associated with rheumatologic disease), and (3) normal NK cell cytolytic activity per cell but NK cell cytopenia (sepsis-induced hyperferritinemic MODS). These different mechanisms are further illustrated in Fig. 14.2.

Absent NK cell cytolytic activity is the basis for familial HLH, which itself is comprised of a group of monogenic autosomal recessive or $\mathrm{X}$-linked primary immune deficiency diseases characterized by the absence of crucial components of the perforin-granzyme pathway needed for NK cells to kill viruses and induce apoptosis in cancer and host inflammatory cells. Gene knockout models for this pathway result in murine HLH and death after infection with otherwise innocuous LCM (lymphocytic choriomeningitis) virus infection. In the wild-type mouse exposed to LCM virus infection, host $\mathrm{NK}$ and $\mathrm{T}$ cells respond to control the virus, whereas in the perforin knockout mouse, LCM virus infection cannot be killed leading to T-cell proliferation and activation and consequent overproduction of interferon- $\gamma$ (reflected by very high levels of CXCL9, the monokine induced by interferon- $\gamma$ ). T lymphocyte-derived interferon- $\gamma$ induces macrophage activation and organ injury. Etoposide, one of the traditional treatments for familial HLH, destroys proliferating activated $\mathrm{T}$ cells and reduces interferon- $\gamma$ production, preventing undue macrophage activation.

Reduced NK cytolytic activity is the basis of rheumatologic or drug-induced MAS. For instance, some patients with systemic juvenile idiopathic arthritis (sJIA), a known trigger for 


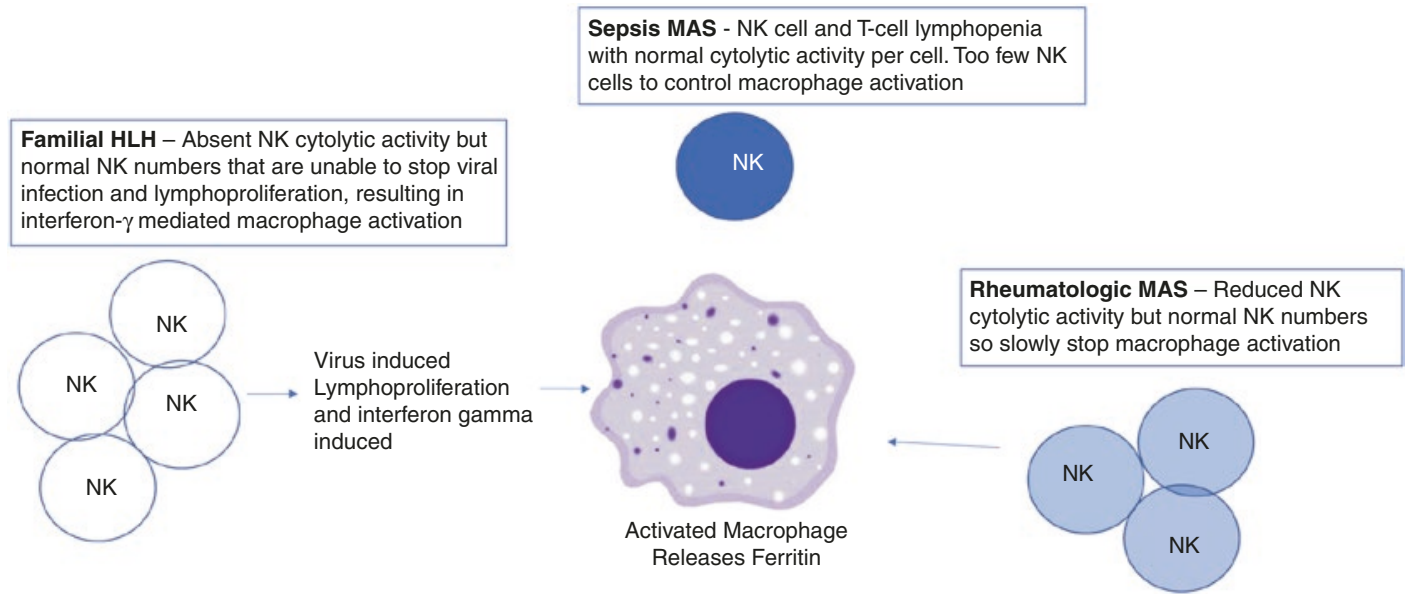

Fig. 14.2 Different natural killer (NK) cell pathobiologies: familial hemophagocytic lymphohistiocytosis (HLH) (white NK cells = absent cytolytic activity); rheumatologic disease-related macrophage activation syndrome (MAS)

sHLH, are hypomorphic or heterozygotes for the perforin-granzyme pathway gene variants. As heterozygotes, these patients have some NK cytolytic activity. For the most part, these patients respond nicely to anti-inflammatory therapies such as anakinra (IL-1 receptor antagonist protein). The IL-1 receptor antagonist protein anakinra is FDA approved for inflammasome-driven conditions (e.g., sJIA). IL-18, the interferon- $\gamma$-inducible factor, is also increased in these patients. There is optimism that IL-18-binding protein (which neutralizes IL-18) as well as interferon- $\gamma$ monoclonal antibody could help these patients.

Reduced number of NK cells with normal NK cytolytic activity per cell is the basis of hyperferritinemic sepsis-induced MODS. When NK cell numbers recover, then inflammation and MODS resolve. During bacterial infection, NK cells switch from an overall low cytokine-producing, high cytolytic activity phenotype to a high cytokine-producing, low cytolytic activity phenotype [13]. In hyperferritinemic sepsis-induced MODS, NK cell cytopenia and T-cell cytopenia occur with reticuloendothelial system activation but decreased to absent interferon- $\gamma$ production. When the reduction in NK cell and T-cell numbers is below $10 \%$ of normal, there is a significant decrease in host ability to kill viruses and (light blue NK cells = reduced cytolytic activity); and hyperferritinemic sepsis-induced multiple-organ dysfunction syndrome (MODS) (dark blue NK cells = low numbers of NK cells with normal cytolytic activity)

cancer cells as well as to induce apoptosis in activated macrophages. Because $\mathrm{T}$ cells and interferon-gamma production are already low to absent, etoposide is unlikely to be of benefit in reducing macrophage activation in these children. Indeed, etoposide may worsen outcomes in sepsis patients by preventing the recovery of lymphocyte counts needed to resolve infection. Compared to patients with other forms of HLH, those with hyperferritinemic sepsisinduced MODS have lower production of interferon- $\boldsymbol{\gamma}$-inducing IL-18. The promise of interferon- $\gamma$ monoclonal antibodies in treating familial HLH and MAS is therefore less likely to be realized in hyperferritinemic sepsis-induced MODS. Further, inability to produce interferon- $\gamma$ is associated with increased mortality in experimental models of sepsis.

Two interferon- $\gamma$-independent pathways that induce macrophage activation-related hyperferritinemia in patients with sepsis-induced MODS are free hemoglobin and DNA viremia. These pathways are depicted in Fig. 14.3 [14]. Endotheliopathy in sepsis leads to hemolysis, particularly in patients with gene variants related to atypical hemolytic uremic syndrome (HUS) and low to absent inhibitory complement production. The released free hemoglobin complexes 
Hemolysis leads to hemoglobin haptoglobin complexes which activate macrophages through CD163 receptors to produce ferritin
DNA viruses complex with TLR9 activating the inflammasome, which in turns generates IL-1 and IL-18 which further activate the inflammasome

Activated macrophage produces ferritin, which increases innate inflammation that induces IL-1

mediated liver injury while also suppressing adaptive immunity and host ability to quell viral infection

Fig. 14.3 Interferon- $\gamma$-independent pathways to macrophage activation during sepsis include free hemoglobin from hemolysis or blood transfusions, as well TLR9 stimulation from DNA viral reactivation

with haptoglobin and binds to the macrophage CD163 receptor, which is internalized, leading to the production and release of extracellular ferritin. Extracellular ferritin activates liver stellate cells causing pro-inflammatory cytokinemediated liver injury. Ferritin also increases tolllike receptor (TLR) expression including TLR9 on innate immune cells while inhibiting the adaptive immune response by preventing lymphopoiesis. DNA viremia, enabled in part by lymphopenia-induced viral reactivation, complexes with the macrophage TLR9 receptor resulting in inflammasome activation and production of IL-1, IL-18, and more extracellular ferritin. This cascade results in a feed-forward positive feedback inflammation loop with more liver injury, innate immune cell inflammation, and adaptive immune cell depression [14]. Plasma exchange can be used in these patients to remove free hemoglobin and extracellular ferritin as well as to replace inhibitory complement. Intravenous immunoglobulin (IVIg) can also be given to neutralize DNA viremia and to block further TLR9 stimulation. Interestingly, IL-1 receptor antagonist protein is effective in reversing both ferritin and TLR9-induced liver injury while inducing a delayed type 1 interferon response to combat DNA viremia.

\section{Diagnosis and Therapeutic Options for Secondary HLH}

Despite different pathobiologies, familial HLH and both forms of sHLH - MAS induced by rheumatologic disease and hyperferritinemic sepsis-induced MODS - are all characterized by uncontrolled macrophage activation and are traditionally diagnosed by the presence of five of eight clinical criteria that include ferritin $>500 \mathrm{ng} / \mathrm{ml}$, two-line cytopenia, organomegaly, hypertriglyceridemia, hypofibrinogenemia, elevated soluble CD25, absent natural killer (NK) cytotoxic activity, and hemophagocytosis (Fig. 14.1). Importantly, though these criteria were initially created to diagnose familial HLH, there are some clinical data supporting approaches to the treatment of sHLH using this diagnostic methodology [4-7]. Additionally, Demirkol et al. evaluated different therapies for Turkish children who met criteria for sHLH in a cohort study [15]. They excluded children who were under 2 years, had a history of consanguineous parenting, or had a previous young family member who died from fever because these children were more likely to have familial HLH. Mortality in these excluded children, who were treated by hematologists with etoposide and dexamethasone, was 50\%. All the included 
children (i.e., without these familial HLH risk factors) were diagnosed with sHLH and had five of the aforementioned eight clinical criteria and had five to six organ failures. Centers in one treatment cohort administered the familial HLH protocol of dexamethasone and/or etoposide along with daily plasma exchange to these children with SHLH and observed a $50 \%$ mortality rate, whereas centers in the other treatment cohort administered a less immune suppressive regimen of methylprednisolone with or without intravenous immunoglobulin (IVIG) with daily plasma exchange and observed a $0 \%$ mortality rate. Based on the above data, though it may be appropriate to use the diagnostic criteria for familial HLH to diagnose sHLH, the traditional familial HLH protocol of dexamethasone and etoposide should be replaced by less toxic treatment strategies for patients with sHLH.

\section{Clinical Vignette 2: sHLH Induced by}

\section{Systemic Juvenile Arthritis}

A febrile 7-year-old child presents to an ICU with rash, leukocytosis, arthritis, and laboratory data concerning for $\mathrm{SHLH}$. The intensivist calls the rheumatologist, who believes the presentation is consistent with systemic juvenile arthritis-related MAS. She recommends treatment with methylprednisolone and the $I L-1$ receptor antagonist anakinra. Other laboratory testing is sent to rule out systemic lupus erythematosus, sarcoidosis, scleroderma, Sjogren's syndrome, and Kawasaki's disease.

Patients with autoimmune rheumatologic disease have increased inflammasome activation and reduced NK activity without lymphoproliferation. Accordingly, rather than etoposide and dexamethasone regimens, methylprednisolone and anakinra are typically recommended to control inflammasome activation associated with rheumatologic disease. Other biologics and chemotherapeutic regimens, however, such as cyclophosphamide, methotrexate, tocilizumab, or etoposide as well as plasma exchange, are considered if the patient remains recalcitrant.
Because three of the eight clinical criteria (e.g., soluble CD25 levels, NK cytotoxicity, and hemophagocytosis) used to identify patients with HLH are not easily accessible tests, rheumatologists have sought to redefine sHLH with other criteria sets that use more readily available laboratory tests. For example, utilizing current literature, Ravelli and colleagues have provided a consensus statement defining sHLH in a child with known systemic juvenile idiopathic arthritis (sJIA), a common trigger of macrophage activation, based on the presence of fever, ferritin $>684 \mathrm{ng} / \mathrm{mL}$, and any two of the following: platelet count $<181 \mathrm{~K}$, ALT $>48 \mathrm{IU} / \mathrm{L}$, triglycerides $>156 \mathrm{mg} / \mathrm{dL}$, and fibrinogen $<360 \mathrm{mg} / \mathrm{dL}$ [16]. Though recommended for patients with MAS secondary to sJIA, these criteria can be applied to patients with MAS secondary to other rheumatologic diseases as well.

Shakoory and colleagues have offered another method for simplifying the diagnosis of and treatment of sHLH [17]. Specifically, they suggested that the combination of hepatobiliary dysfunction and disseminated intravascular coagulation (DIC) can be representative of sHLH including hyperferritinemic sepsisinduced MODS. Further, they hypothesized that if the combination of these two organ dysfunctions represents sHLH, then treatment with IL-1 receptor blockade should improve sepsis-related macrophage activation as it does in the previously mentioned experimental model [10] and in children with sJIA-related MAS [15-17]. In their secondary analysis of an adult with severe sepsis IL-1 receptor blockade trial, Shakoory and colleagues compared patients with combined hepatobiliary dysfunction (HBD) and DIC (HBD + DIC) to those without this combination (nonHBD + DIC) [17]. The investigators found the following: (1) $5.6 \%$ of severe sepsis patients had HBD + DIC; (2) patients with HBD + DIC had a higher incidence of shock $(\mathrm{HBD}+\mathrm{DIC}=95 \%$ versus non$\mathrm{HBD}+\mathrm{DIC}=79 \%)$ and acute kidney injury $(\mathrm{HBD}+\mathrm{DIC}=61 \%$ versus non$\mathrm{HBD}+\mathrm{DIC}=29 \%$ ), but not acute respiratory 
Table 14.1 Simplified clinical criteria used to diagnose rheumatologic or drug-related secondary hemophagocytic lymphohistiocytosis (HLH)/macrophage activation syndrome (MAS) and hyperferritinemic sepsis-induced multipleorgan dysfunction syndrome (MODS)

\begin{tabular}{|c|c|c|}
\hline Familial HLH: five of eight criteria & Rheumatologic/drug-induced MAS & $\begin{array}{l}\text { Hyperferritinemic sepsis-induced } \\
\text { MODS }\end{array}$ \\
\hline $\begin{array}{l}\text { - Fever } \\
\text { - Ferritin > } 500 \text { ng/ml } \\
\text { - Hypertriglyceridemia } \\
\text { - Hypofibrinogenemia } \\
\text { - Two-line cytopenia } \\
\text { - Splenomegaly } \\
\text { - Natural killer cell cytolytic } \\
\text { activity < } 10 \% \\
\text { - Hemophagocytosis } \\
\text { - Elevated soluble CD25 }\end{array}$ & $\begin{array}{l}\text { - Fever } \\
\text { - Ferritin }>684 \mathrm{ng} / \mathrm{mL} \\
\text { - With any two of the following: } \\
\text { Platelet count }<181,000 \\
\text { Alanine transferase }>48 \mathrm{IU} / \mathrm{L} \\
\text { Triglyceride }>156 \mathrm{mg} / \mathrm{dL} \\
\text { Fibrinogen }<360 \mathrm{mg} / \mathrm{dL}\end{array}$ & $\begin{array}{l}\text { - Hepatobiliary dysfunction } \\
\text { - Disseminated intravascular } \\
\text { coagulation } \\
\text { - +/- ferritin > } 500 \mathrm{ng} / \mathrm{mL}\end{array}$ \\
\hline
\end{tabular}

distress syndrome $(\mathrm{HBD}+\mathrm{DIC}=21 \%$ versus non-HBD + DIC $=26 \%$ ); and (3) IL-1 receptor blockade significantly increased 28-day survival in patients with HBS + DIC (IL-1 blockade $=65.4 \%$ versus placebo $=35 \%$ ), while 28-day survival in non-HBD + DIC did not change (IL-1 blockade $=71 \%$ vs placebo $=71 \%$ ). It can be, therefore, extrapolated that sHLH diagnosed by the presence of HBD and DIC constitutes a small but significant subset among severe sepsis patients; death attributable to superimposed SHLH can be remedied in some patients with adjunctive therapy with IL-1 receptor blockade.

More recently, Hellenic and Swedish investigators reported outcomes in adult patients with hyperferritinemic sepsis-induced MODS, though they described these patients as having sepsis and concomitant "macrophage activation-like syndrome (MALS)" [18]. In this study, they found that the degree of hyperferritinemia was most predictive of mortality. As a result, they concluded that the presence of hyperferritinemia (ferritin $>500 \mathrm{ng} / \mathrm{dL}$ ), hepatobiliary dysfunction, and disseminated intravascular coagulation can be used to diagnose sHLH and feasibly be used to identify patients with sHLH or hyperferritinemic sepsisinduced MODS for "early clinical trials" of inflammation-modulating therapies $[5-7,15$, 17, 18]. The simplified approaches to the diagnosis of MAS and hyperferritinemic sepsis-induced MODS are summarized in Table 14.1.

\section{Clinical Vignette 3: Hyperferritinemic}

\section{Sepsis-Induced MODS}

A 3-year-old patient is admitted to the ICU with a presumed diagnosis of sepsis. Antibiotics and source control are implemented. Shock, acute respiratory distress syndrome, and acute kidney injury subsequently develop, along with hepatobiliary dysfunction and disseminated intravascular coagulation. Serum ferritin is $1120 \mathrm{ng} /$ $d L$. Diagnosis of hyperferritinemic sepsisinduced multiple-organ dysfunction syndrome (MODS) is made.

An exhaustive search for the diagnosis is also performed, including bacterial sources (mycoplasma, rickettsia, legionella, chlamydia, brucella, and borrelia), fungi and parasites (histoplasmosis, babesia, leishmaniasis, pneumocystis, aspergillus, toxoplasmosis, cryptococcus, and candida), and viruses (EBV, CMV, HSV, HIV, HHV8, HHV6, parvovirus, adenovirus, and influenza) so that appropriate antimicrobial therapy can be ensured.

Plasma exchange, IVIG, and anakinra are considered for treatment. (methylprednisolone can also be considered if the patient does not have a contraindicated infection, e.g., HSV.) In patients with hyperferritinemic sepsis-induced MODS, improvement occurs when NK cell and T lymphocyte counts, which are reduced, recover. 


\section{ICU Management of Secondary HLH for the Pediatric Intensivist}

Patients arriving to the pediatric ICU with a diagnosis of familial HLH will most commonly be receiving etoposide and dexamethasone, while patients arriving with a diagnosis of MAS will be receiving methylprednisolone with biologics such as anakinra (IL-1 receptor antagonist) or tocilizumab (anti-IL-6 antibody). For these children, supportive ICU care is most often necessitated by cardiopulmonary instability or organ failure often associated with HLH. The approach to organ support therapies for these children would be no different for any other child admitted to a pediatric ICU with sepsis, ARDS, and MODS.

More commonly, patients will present to an intensivist without a preceding diagnosis of HLH or MAS but rather with a diagnosis of sepsisinduced MODS. Indeed, most of these patients have sepsis-induced MODS. In addition to standard management for sepsis and multiple-organ failure, intensivists should have a high index of suspicion for sHLH. Though some centers have developed multidisciplinary hyperferritinemia interest groups to help the intensivist with this differential diagnosis, most depend on the intensivist to recognize signs and symptoms of this sHLH (Fig. 14.1) and call for specialty consultation. Of note, history of consanguineous parenting or family history of children dying from fever, especially in a patient presenting less than 2 years of age with significant CNS pathology, should raise suspicion for familial HLH. For patients with subacute arthralgia or arthritis, rheumatologic disease with MAS should be considered, although sHLH in patients with septic arthritis will be more likely to be hyperferritinemic sepsis-induced MODS.

For any patient presenting to the pediatric ICU with sepsis, CRP and serum ferritin can be measured on presentation. If ferritin is greater than $500 \mathrm{ng} / \mathrm{dL}$ and other signs or symptoms of sHLH are present, especially DIC or hepatobiliary dysfunction, the diagnosis of hyperferritinemic sepsis-induced MODS should be made. We also recommend monitoring systemic inflammation associated with macrophage activation at the bedside by measuring C-reactive protein (CRP) and serum ferritin at least twice weekly. CRP is a pattern recognition receptor made by the liver in response to bacterial infection or necrotic tissue. $\mathrm{CRP}$ binds to $\mathrm{C}$-components of microbes or the externalized phosphatidylcholine moiety of necrotic cells, complexes with complement, and attaches to the CRP receptor on the macrophage for internalization, degradation, and presentation to the adaptive immune system. Ferritin is released by macrophages in response to free hemoglobin and to DNA viremia. Hyperferritinemia occurs in iron overload states and can also be released by dying cells during necrosis. Mortality risk increases as the circulating CRP and serum ferritin increase. The goal of therapy is to subdue the inflammatory response for which CRP and serum ferritin are surrogate biomarkers. If CRP increases while serum ferritin decreases, this combination is a harbinger of new or worsening infection and warrants attention to better source control and reduction in immune suppression. If ferritin does not come down or increases, then ongoing iron overload and/or macrophage activation (possibly associated with DNA viremia) is likely. Attention should be given to reducing hemolysis, quelling macrophage activation, and neutralizing DNA viremia. This approach to monitoring is illustrated in Fig. 14.4.

As for any patient admitted to an ICU with sepsis, source control and organ support are paramount for patients with hyperferritinemic sepsisinduced MODS. Empiric antibiotics should be started within $1 \mathrm{~h}$ of presentation, and patients should be managed according to current sepsis guidelines [20]. For patients presenting with erythroderma, one must consider toxic shock from Group A Streptococcus or Staphylococcus aureus, which warrants therapy with clindamycin to prevent toxin production and an antimicrobial drug which kills both of these pathogens such as vancomycin. We do not use linezolid because hepatobiliary dysfunction reduces its safety window; specifically, linezolid can become a host mitochondrial toxin when blood concentrations increase due to poor clearance. We recommend immunomodulation with corticosteroids 


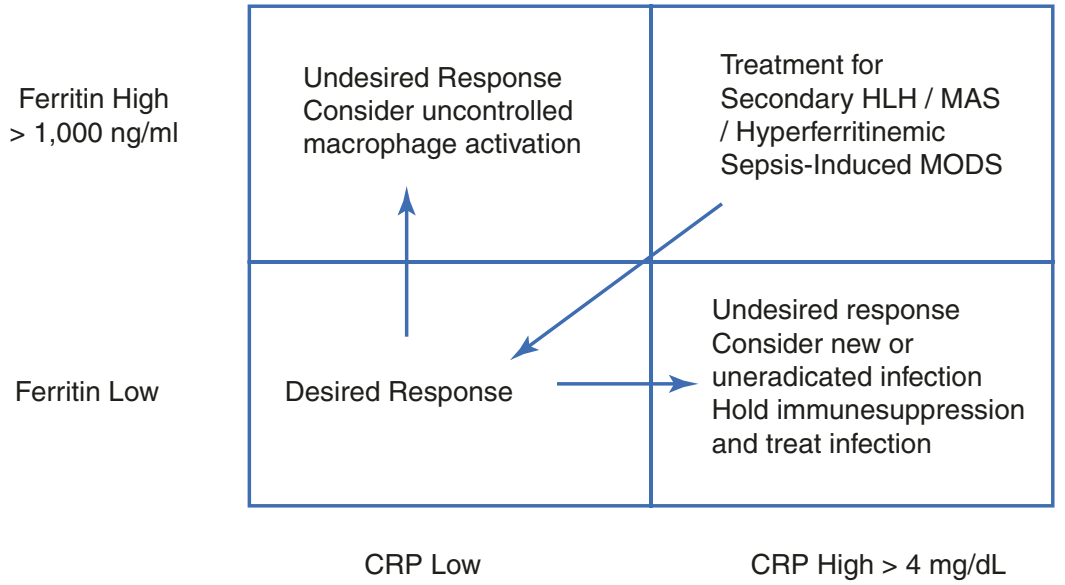

Fig. 14.4 An approach to monitoring inflammation and infection response during initiation of therapy for hemophagocytic lymphohistiocytosis (HLH), macrophage activation syndrome (MAS), and hyperferritinemic sepsis-induced multiple-organ dysfunction syndrome (MODS) by observing changes in $\mathrm{C}$-reactive protein (CRP) and ferritin. C-reactive protein reflects bacterial infection, whereas ferritin reflects macrophage activation

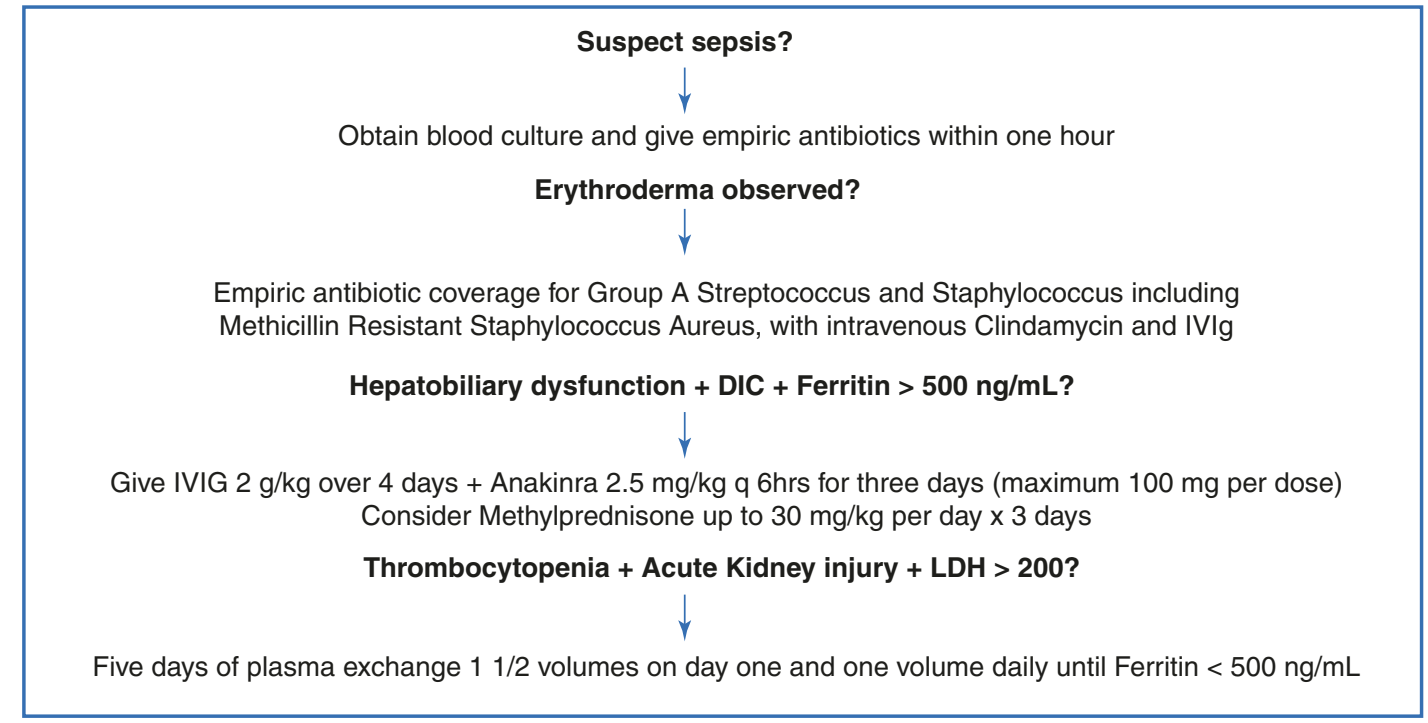

Fig. 14.5 Recommended approach to hyperferritinemic sepsis-induced multiple-organ dysfunction (MODS)

(30 $\mathrm{mg} / \mathrm{kg}$ per day of methylprednisolone $\times$ 3 days) and IVIG ( $2 \mathrm{~g} / \mathrm{kg}$ over $1-4$ days). If the patients have evidence of acute kidney injury, thrombocytopenia, and elevated lactate dehydrogenase, we recommend daily plasma exchange $1.5 \times$ blood volume on day 1 followed by $1 \times$ volume daily until serum ferritin decreases to less than $500 \mathrm{ng} / \mathrm{mL}$. In addition, we recommend the IL-1 antagonist anakinra ( $2.5 \mathrm{mg} / \mathrm{kg}$ q6h to maximum of $100 \mathrm{mg}$ q6h) for 3 days. This treatment pathway is summarized in Fig. 14.5.

\section{Summary and Conclusion}

The prompt recognition and appropriate treatment of familial and secondary HLH present important challenges to clinical intensivist. Differentiating between these clinical scenarios is the first step toward outlining the most appropriate clinical strategies, which is only possible through increasing our understanding of underlying immunopathogenesis that drives the clinical presentation. As our knowledge and 
understanding of the familial and secondary HLH evolve, evidence-based recommendations will better assist the clinical intensivists in their endeavors. Most importantly, a collaborative approach with hematology and rheumatology colleagues is likely the best means of securing a favorable outcome in patients who develop this challenging disease process.

Conflict of Interest Statement On behalf of the authors, there are no conflicts of interest.

\section{References}

1. Castillo L, Carcillo J. Secondary hemophagocytic lymphohistiocytosis and severe sepsis/ systemic inflammatory response syndrome/multiorgan dysfunction syndrome/macrophage activation syndrome share common intermediate phenotypes on a spectrum of inflammation. Pediatr Crit Care Med. 2009; 10:387-92.

2. Suster S, Hilsenbeck S, Rywlin AM. Reactive histiocytic hyperplasia with hemophagocytosis in hematopoietic organs: a reevaluation of the benign hemophagocytic proliferations. Hum Pathol. 1988;19:705-12.

3. Strauss R, Neureiter D, Westenburger B, Wehler M, Kirchner T, Hahn EG. Multifactorial risk analysis of bone marrow histiocytic hyperplasia with hemophagocytosis in critically ill medical patients-a postmortem clinicopathologic analysis. Crit Care Med. 2004;32(6):1316-21.

4. Inai $\mathrm{K}$, Noriki S, Iwasaki H, Naiki H. Risk factor analysis for bone marrow histiocytic hyperplasia with hemophagocytosis: an autopsy study. Virchows Arch. 2014;465:109-18.

5. Buyse S, Teixeira L, Galicier L, Mariotte E, Lemiale V, Seguin A, et al. Critical care management of patients with hemophagocytic lymphohistiocytosis. Intensive Care Med. 2010;36:1695-702.

6. Créput C, Galicier L, Buyse S, Azoulay E. Understanding organ dysfunction in hemophagocytic lymphohistiocytosis. Intensive Care Med. 2008;34:1177-87.

7. Emmenegger U, Schaer DJ, Larroche C, Neftel KA. Haemophagocytic syndromes in adults: current concepts and challenges ahead. Swiss Med Wkly. 2005; 135:299-314.

8. Steinberg S, Flynn W, Kelley K, Bitzer L, Sharma P, Gutierrez C, et al. Development of a bacteria-independent model of the multiple organ failure syndrome. Arch Surg. 1989;124:1390-5.

9. Behrens EM, Canna SW, Slade K, Rao S, Kreiger PA, Paessler M, et al. Repeated TLR9 stimulation results in macrophage activation syndrome-like disease in mice. J Clin Investig. 2011;121:2264-77.

10. Tsujimoto H, Ono S, Matsumoto A, Kawabata T, Kinoshita M, Majima T, et al. A critical role of $\mathrm{CpG}$ motifs in a murine peritonitis model by their binding to highly expressed toll-like receptor-9 on liver NKT cells. J Hepatol. 2006;45:836-43.

11. Petrasek J, Dolganiuc A, Csak T, Kurt-Jones EA, Szabo G. Type I interferons protect from Toll-like receptor 9-associated liver injury and regulate IL-1 receptor antagonist in mice. Gastroenterology. 2011;140:697-708.

12. Szabo G, Petrasek J. Inflammasome activation and function in liver disease. Nat Rev Gastroenterol Hepatol. 2015;12:387-400.

13. Halstead ES, Carcillo JA, Schilling B, Greiner RJ, Whiteside TL. Reduced frequency of CD56 dim CD16 pos natural killer cells in pediatric systemic inflammatory response syndrome/sepsis patients. Pediatr Res. 2013;74:427-32.

14. Kernan KF, Carcillo JA. Hyperferritinemia and inflammation. Int Immunol. 2017;29:401-9.

15. Demirkol D, Yildizdas D, Bayrakci B, Karapinar B, Kendirli T, Koroglu TF, et al. Hyperferritinemia in the critically ill child with secondary hemophagocytic lymphohistiocytosis/sepsis/multiple organ dysfunction syndrome/macrophage activation syndrome: what is the treatment? Crit Care. 2012;12(16):R52.

16. Ravelli A, Minoia F, Davì S, Horne A, Bovis F, Pistorio A, et al. Classification criteria for macrophage activation syndrome complicating systemic juvenile idiopathic arthritis: a European league against rheumatism/American college of rheumatology/ Paediatric rheumatology international trials organisation collaborative initiative. Arthritis Rheumatol. 2016;68:566-76.

17. Shakoory B, Carcillo JA, Chatham WW, Amdur RL, Zhao H, Dinarello CA, et al. Interleukin-1 receptor blockade is associated with reduced mortality in Sepsis patients with features of macrophage activation syndrome: reanalysis of a prior phase III trial. Crit Care Med. 2016;44:275-81.

18. Rajasekaran S, Kruse K, Kovey K, Davis AT, Hassan NE, Ndika AN, et al. Therapeutic role of anakinra, an interleukin-1 receptor antagonist, in the management of secondary hemophagocytic lymphohistiocytosis/ sepsis/multiple organ dysfunction/macrophage activating syndrome in critically ill children. Pediatr Crit Care Med. 2014;15:401-8.

19. Kyriazopoulou E, Leventogiannis K, Norrby-Teglund A, Dimopoulos G, Pantazi A, Orfanos SE, et al. Macrophage activation-like syndrome: an immunological entity associated with rapid progression to death in sepsis. BMC Med. 2017;18(15):172.

20. Rhodes A, Evans LE, Alhazzani W, Levy MM, Antonelli M, Ferrer R, et al. Surviving sepsis campaign: international guidelines for management of sepsis and septic shock 2016. Crit Care Med. 2016;45:486-552. 